\title{
ESAT-6: a perfect warfare or a weak link in armor
}

\author{
Volume 5 Issue I - 2017
}

\author{
Sachin Kumar Samuchiwal \\ Department of Medicine, Harvard Medical School, USA
}

Keywords: tuberculosis, BCG, $m . t b$ virulence, ESAT-6, immune response, IFN- $\gamma, \mathrm{TB}, \mathrm{TNF}-\alpha$

Abbreviations: $\mathrm{TB}$, tuberculosis; $\mathrm{rBCG}$, recombinant $\mathrm{BCG}$; $\mathrm{RD} 1$, region of difference 1; TNFR1, TNF- $\alpha$ receptor1; SOD, superoxide dismutase; ICA, immunocytochemical assays

\section{Editorial}

Tuberculosis (TB) an ancient dreaded disease remains second leading cause of death from an infectious disease worldwide. One third of global population is latently infected with TB and it kills millions each year. ${ }^{1}$ To make the matter worse, currently available vaccine BCG provides limited protection against pulmonary TB in adults. BCG induces effective Th1 response, but fails to induce Th17 response in the lung, a factor mostly responsible for the substandard efficacy of BCG. ${ }^{2}$ Considerable efforts are being made to generate recombinant $\mathrm{BCG}(\mathrm{rBCG})$ but with little headway. Causative agent of TB Mycobacterium tuberculosis (M.tb) is remarkably skillful in eluding host defense and modulating immune response for its own benefits. An interesting approach for combating TB is to target $M$. $t b$ virulence factors. ${ }^{3}$ One such key protein is ESAT-6, encoded by region of difference 1 (RD1), the region absent from BCG and most attenuated mycobacterial strains. ${ }^{4,5}$

Role of ESAT-6 in M.tb virulence has been highlighted in this editorial. ESAT-6 possesses membrane lytic activity due to its helixturn-helix structure and hydrophobic nature, an attribute that helps $M$. $t b$ escape phagolysosome, cell- to-cell spread and dissemination. ESAT-6 can alter host defense by decreasing MHC-II expression on macrophages, granuloma formation and decreasing ROS production to aid in intramacrophagial M.tb survival. ${ }^{6}$ ESAT-6 or CFP-10/ESAT6 complex is also known to attenuate host innate immune response by inhibiting production of IL-12, TNF- $\alpha$, and apoptosis inhibition in M.tb infected macrophages to achieve greater vial load and cell-cell spread. ${ }^{7,8}$ ESAT- 6 is also supposed to bind to T-cell surface and belittles T-cell immunity by inhibiting T-cell proliferation and activation. ${ }^{9}$ As we know the production of the proinflammatory cytokine TNF- $\alpha$ is critical for controlling M.tb infection as it induces apoptosis via TNF- $\alpha$ receptor1 (TNFR1) signaling. Therefore ESAT- 6 and CFP10/ESAT-6 complex inhibit apoptosis of infected cells by decreasing TNF- $\alpha$ production to attain bacterial buildup and dissemination. There is a large body of literature suggesting that $M . t b$ virulence and modulation of host defense in an intricate communication of M.tb and host proteins, and targeting these interactions can offer a pivotal tool in fight against TB. ESAT-6 has been gaining more and more popularity over the time as one such candidate to be targeted for antitubercular therapeutics development.

Widespread efforts are being made to improve current BCG vaccine or to develop new TB vaccines. An in trend approach is to develop efficacious vaccines by combining ESAT-6 with other M.tb virulence determining proteins. Several $\mathrm{rBCG}$ strains have been created that express ESAT-6 fusion proteins along with other M.tb proteins like Ag85B, Rv3620c, TNF- and Rv2608, complement RD1, or combine
ESAT-6 with Antigen 85 family and superoxide dismutase (SOD), interestingly these new rBCG vaccines demonstrate a strong T-cell response and high expression of Th1 cytokines in experimental animals. ${ }^{10,11}$ Even chimeric DNA vaccines are also underway and one such example is developed by combining ESAT-6 gene from M.tb and kinesin motor domain gene of Leishmania donovani, which resulted in robust IFN- $\gamma$ and IL-2 production in mice. While another nanoparticle based chimeric DNA vaccine contains Ag85A, ESAT-6 and IL-21 construct. ${ }^{11}$ On another front, an strong ESAT- 6 binding peptide when expressed in $H 37 R v$ strains, it disintegrates bacterial cell wall and inhibits extracellular as well as intracellular mycobacterial growth by inducing both Th1 and Th17 host protective responses. ${ }^{2}$ A significant number of adenoviral and subunit vaccines are also exploiting the importance of ESAT-6 in M.tb virulence. A subunit vaccine based on a fusion protein between Ag85B and ESAT-6 has shown efficient longterm memory immunity induction that is highly protective against TB. ${ }^{11}$ Ongoing work on numerous ESAT- 6 amalgamated TB vaccines, may lay the foundation for developing new and realistic alternative vaccines to $B C G$ in the near future.

As ESAT-6 has already established itself as a crucial protein during the havoc raised by M.tb in host system, it also confers strong antigenic properties that lead to various responses useful for $M$. $t b$ diagnosis. It is known that human effector memory T-cells produce large amounts of IFN- $\gamma$ in response to ESAT-6, and not so long ago 2 ex vivo IFN- $\gamma$ release assays (IGRAs) were developed that utilize this response to diagnose latent and active tuberculosis infection. ${ }^{12} \mathrm{~A}$ number of poly-proteins have been developed that comprises ESAT6/RD1 proteins in addition to other M.tb proteins and indirect ELISA assays based on these constructs have shown higher sensitivity and specificity for serodiagnosis of TB. One such study uses recombinant proteins ESAT6, CFP10, Ag85B and Hsp16.3 alone or in combination to diagnose active and latent TB infections ${ }^{13}$ There are also aptamerbased methods for ESAT- 6 and CFP-10 detection in human patient samples for diagnosis of pulmonary and extrapulmonary TB. In addition, immunocytochemical assays (ICA) that detect ESAT-6 and CFP-10 are reported to be less expensive and more effective TB tests, which could be useful in high TB burden third world countries. Both ESAT-6 and CFP-10 proteins, used as recombinant antigens, overlapping synthetic peptides or single immunodominant peptides, have repeatedly proven to be useful in the specific diagnosis of active 
and latent TB and monitoring the efficacy of chemotherapy against TB using (IGRAs).

The RD1 coded ESAT-6; almost unique to virulent mycobacterial strains is a crucial protein in M.tb infection and dissemination due to its structure, physicochemical properties and ability to module host immune response in favor of the pathogen. Interestingly on the flip side ESAT-6 provides a multifaceted target protein that is ideal for vaccine and diagnostics development. As efforts towards development of new strategies for combating TB is underway on global scale, ESAT-6/ $\mathrm{RD} 1$ might prove to be a chink in the armor of M.tb, hopefully leading to eventual eradication of this ancient dreaded scourge.

\section{Acknowledgments}

None.

\section{Conflicts of interest}

Author declares there are no conflicts of interest.

\section{Funding}

None.

\section{References}

1. Tuberculosis (TB ). Global tuberculosis report 2016. World Health Organization. 2016.

2. Samuchiwal SK, Tousif S, Singh DK, et al. A peptide fragment from the human COX3 protein disrupts association of Mycobacterium tuberculosis virulence proteins ESAT-6 and CFP10, inhibits mycobacterial growth and mounts protective immune response. $B M C$ Infect Dis. 2014;14:355.

3. Alksne LE, Projan SJ. Bacterial virulence as a target for antimicrobial chemotherapy. Curr Opin Biotechnol . 2000;11(6):625-636.
4. Gordon SV, Brosch R, Billault A, et al. Identification of variable regions in the genomes of tubercle bacilli using bacterial artificial chromosome arrays. Mol Microbiol. 1999;32(3):643-655.

5. Mahairas GG, Sabo PJ, Hickey MJ, et al. Molecular analysis of genetic differences between Mycobacterium bovis BCG and virulent M. bovis. J Bacteriol. 1996;178(5):1274-1282.

6. Samuchiwal SK, Tousif S, Singh DK, et al. A novel peptide interferes with Mycobacterium tuberculosis virulence and survival. FEBS Open Bio . 2014;4:735-740.

7. Pathak SSK, Basu S, Basu KK, et al. Direct extracellular interaction between the early secreted antigen ESAT-6 of Mycobacterium tuberculosis and TLR2 inhibits TLR signaling in macrophages. Nat Immunol. 2007;8(6):610-618.

8. Guo S, Xue R, Li Y, et al. The CFP10/ESAT6 complex of Mycobacterium tuberculosis may function as a regulator of macrophage cell death at different stages of tuberculosis infection. Med Hypotheses. 2012;78(3):389-392.

9. Barnes PF, Samten B, Shams H, et al. Progress in understanding the human immune responses to Mycobacterium tuberculosis. Tuberculosis (Edinb) . 2009;89(Suppl 1):S5-S9.

10. Tyagi AK, Nangpal P, Satchidanandam V. Development of vaccines against tuberculosis . 2007;91(5):469-478.

11. Gupta UD, Katoch VM, McMurray DN. Current status of TB vaccines. Vaccine. 2007;25(19):3742-351.

12. Menzies D, Pai M, Comstock G. Meta-analysis: new tests for the diagnosis of latent tuberculosis infection: areas of uncertainty and recommendations for research. Ann Intern Med . 2007;146(5):340-354.

13. Zhang C, Song X, Zhao Y, et al. Mycobacterium tuberculosis Secreted Proteins As Potential Biomarkers for the Diagnosis of Active Tuberculosis and Latent Tuberculosis Infection. J Clin Lab Anal. 2015;29(5):375-382. 